\title{
Linx
}

Revue des linguistes de l'université Paris X Nanterre

12 | 2002

«Comme la lettre dit la vie »

\section{Histoire et parole dans la «Vie de Saint Louis » de Joinville}

Roberte Tomassone

\section{OpenEdition}

Journals

Édition électronique

URL : http://journals.openedition.org/linx/1318

DOI : $10.4000 /$ linx. 1318

ISSN : 2118-9692

Éditeur

Presses universitaires de Paris Nanterre

Édition imprimée

Date de publication : 1 octobre 2002

Pagination : 269-275

ISSN : 0246-8743

\section{Référence électronique}

Roberte Tomassone, « Histoire et parole dans la « Vie de Saint Louis » de Joinville », Linx [En ligne],

12 | 2002, mis en ligne le 10 octobre 2012, consulté le 19 avril 2019. URL : http://

journals.openedition.org/linx/1318; DOI : 10.4000/linx.1318

Ce document a été généré automatiquement le 19 avril 2019.

Département de Sciences du langage, Université Paris Ouest 


\title{
Histoire et parole dans la «Vie de Saint Louis » de Joinville
}

\author{
Roberte Tomassone
}

1 Que dire de nouveau sur la vie de Saint Louis ? Et que dire de plus de « La vie de Saint Louis »?

2 Joinville, «témoin exceptionnel ", selon Jacques Le Goff : Joinville, qui fait souvent dire à l'historien «Ah oui ! cela, c'est bien le 'vrai' Saint Louis! »'. Mais Joinville est aussi le premier, écrivant en français, à parler de lui à la première personne ${ }^{2}$.

3 A lire Joinville, on se demande quel a été - consciemment ou inconsciemment - son objet : le roi ou lui-même ? S'agit-il d'une biographie ou d'une autobiographie ${ }^{3}$ ?

4 Joinville historien? narrateur ? témoin? L'observation d'indices textuels et énonciatifs, dans le récit de la «croisade d’Egypte » (\$132 à 310$)$, peut apporter à ces questions quelques éléments de réponse.

\section{Joinville historien : la construction de l'histoire}

5 Le récit de Joinville est, selon la tradition, divisé en paragraphes et alinéas. La construction thématique du récit, complexe, la répartition des thèmes et des rhèmes, les thématisations, organisent et hiérarchisent la présentation des faits.

6 Le schéma le plus fréquent est constitué par la thématisation d'un circonstant, temporel le plus souvent, quelquefois spatial : sans doute est-ce le schéma que l'on s'attend à rencontrer dans ce type de texte, où les repères chronologiques sont essentiels.

7 Chronologie absolue : Maintenant que mars entra (\$ 146); Le samedi ...; L'andemain de la Penthecouste ..., Le jeudi après Penthecouste (§ 148); Quant la Saint Remy fu passee (\$180); En l'entré des Advens (§ 184); Le jour de la Saint Nicholas (§ 185); Le jour de Noel (§ 196); La vegile de quaresme pernant ( $\$ 297)$; etc.

8 La thématisation de ces circonstants assure le cadrage thématique du récit, reliant les faits au calendrier, et pose des jalons à partir desquels se déroulent les épisodes, eux aussi 
encadrés et jalonnés par la thématisation de circonstants temporels : En ce point que le roy sejournoit en Cypre ( $(133)$; En ce point que nous sejournames en Cypre..., Quant nous venimes la (\$137); L'endemain ( $(138)$; Après ce que nous feumes arivés en Egypte (\$ 140); En ce point que nous venimes en Cypre ( $\$ 141)$; Quant vint au vendredi (\$151); Quant nos gens virent que nous n'ariens point de galie... ( $\$ 152)$; Endementres que je menoie ses gens (\$153); Si tost comme il nous virent a terre ( $\$ 156)$, etc. La liste n'est évidemment pas exhaustive ! La thématisation de ces indicateurs temporels relatifs met en place une chronologie détaillée, explique et justifie l'enchaînement des faits ( $\$ 152)$, tantôt fait progresser le récit (\$ 156) et tantôt le retarde, arrêtant le cours des événements sur un épisode concomitant ( $\$ 133$ et 137), un retour en arrière, ou quelque commentaire (Dans cette bataille, § 246).

Ainsi, le procédé, constant, de thématisation des circonstants, qui peut apparaître en première lecture comme un procédé d'écriture systématique et répétitif, architecture de fait un récit souple et varié, qui progresse à des rythmes divers, par à-coups, ruptures et retours.

A l'intérieur des unités textuelles, de dimensions diverses, ainsi délimitées, s'organisent d'autres types de schémas thématiques et de progressions. Nous en donnerons comme exemple les paragraphes 222 à 228 .

222 Quant moy et mes chevaliers venimes hors de l'ost aus Sarrazins, nous trouvames bien . $\mathrm{VI}^{\mathrm{M}}$. Turs, par esme, qui avoient lessiees leur herberges et se estoient trait aus chans. Quant il nous virent, il nous vindrent sus courre, et occistrent mon seigneur Hugue de Trichastel, seigneur de Conflans, qui estoit avec moy a baniere. Moy et mes chevaliers ferimes des esperons et alames rescourre mon seigneur Raoul de Wanou, qui estoit avec moy, que il avoit tiré a terre. 223 Endementieres que je en revenoie, les Turs m'apuierent de leurs glaives. Mon cheval s'agenoilla pour le fez que il senti, et je en alé outre parmi les oreilles du cheval ; et me resdresçai au plus tos que je peu, mon escu a mon col et m'espee en ma main. Et mon seigneur Erart de Severey, que Dieu absoille, qui estoit entour moy, vint a moy et nous dit que nous nous treissions emprés une meson deffaite, et illec attenderions le roy qui venoit. Ainsi comme nous en alions a pié et a cheval, une grant route de Turs vint hurter a nous et me porterent a terre, et alerent par dessus moy et volerent mon escu de mon col. 224 Et quant il furent outre passez, mon seigneur Erart de Syverey revint sur moy et m'enmena, et en alames jusques aus murs de la meson deffete ; et illec revindrent a nous mon seigneur Hugues d'Escoz, mon seigneur Ferri de Loupey, mon seigneur Renaut de Menoncourt. Illec, les Turs nous assailloient de toutes pars ; une partie d'eulz entrerent en la meson deffete et nous piquoient de leur glaives par desus. Lors me dirent mes chevaliers que je les preisse par les frains, et je si fis, pour ce que les chevaus ne s'enfouissent. Et il se deffendoient des Turs si viguereusement car il furent loez de touz les preudommes de l'ost, et de ceulz qui virent le fait et de ceulz qui l'oïrent dire. 225 La fu navré mon seigneur Hugue d'Escos de .III. glaives ou visage, et mon seigneur Raoul et mon seigneur Ferri de Loupey d'un glaive parmi les espaules; et fu la plaie si large que le sanc li venoit du cors aussi come le bondon d'un tonnel. Mon seigneur Erart de Siverey fu feru d'une espee parmi le visage, si que le nez li cheoit sur le levre. Et lors il me souvint de mon seigneur saint Jaque : « Biau sire saint Jaque, que j'ai requis, aidiés moy et secourez a ce besoing ! ». 226 Maintenant que j'oi faite ma priere, mon seigneur Erart de Siverey me dit : « Sire, se vous cuidiés que moy ne mes hers 
n'eussions reprouvier, je vous iroie querre secours au conte d'Anjou que je voi la enmi les chans. » Et je li dis : « Mesire Erart, il me semble que vous feriés vostre grant honeur se vous nous aliés querre aide pour nos vies sauver, car la vostre est bien en avanture. » Et je disoie bien voir, car il fu mort de celle bleceure. Il demanda conseil a touz nos chevaliers qui la estoient, et touz li louerent ce que je li avoie loé. Et quant il ö̈ ce, il me pria que je li lessasse aller son cheval, que je li tenoie par le frain avec les autres, et je si fiz. 227 Au conte d'Anjou vint et li requist que il me venist secourre moy et mes chevaliers. Un riche homme qui estoit avec li li desloa, et le conte d'Anjou li dit que il feroit ce que mon chevalier li requeroit. Son frain tourna pour nous venir aidier, et pluseurs de ses serjans ferirent des esperons. Quant les Sarrazins les virent, si nous lessierent. Devant ces sergans vint mon seigneur Pierre de Alberive, l'espee ou poing, et quant il veit que les Sarrazins nous eurent lessiés , il courut sur tout plein de Sarrazins qui tenoient mon seigneur Raoul de Vaunou et le rescoÿ moult blecié.

Comme dans l'ensemble $\mathrm{du}$ texte, les circonstants temporels assurent le cadrage thématique, en inscrivant le récit dans la succession chronologique. Des jalons "principaux», constitués par la thématisation de subordonnées temporelles, et coïncidant souvent avec l'amorce d'un nouveau paragraphe (voir texte ci-dessus), délimitent des unités textuelles qui segmentent le récit en épisodes, en séquences dont l'unité interne est assurée en partie par les enchaînements thématiques - progression linéaire dans le paragraphe 222; thème constant, paragraphe 223 et 226 - et, paradoxalement, les ruptures thématiques qui font, si l'on ose ce parallèle anachronique, comme un déplacement de caméra d'un personnage à l'autre; ainsi, par exemple, au paragraphe 223 :

Endementieres que je en revenoie, ...

Mon cheval ...

Et mon seigneur Erart de Severey, .....

Ainsi comme nous en alions a pié et a cheval, ...

12 Le paragraphe 224, acmè de la bataille, offre la synthèse de ces procédés de structuration du texte: accélération de l'action dans une succession de phrases presque toutes en rupture thématique et cadrées dans la thématisation de circonstants adverbiaux :

\begin{tabular}{|l|l|l|}
\hline & Et quant il furent outrepassez... & mon seigneur Erart de Siverey \\
\hline Et illec...... & mon seigneur Hugues d'Escoz \\
\hline & & mon seigneur Ferri de Loupey \\
\hline & Illec & mon seigneur Renaut de Menoncourt \\
\hline & Une partie d'eulx & les Turs \\
\hline & Lors me dirent mes chevaliers & \\
\hline & et je & \\
\hline
\end{tabular}


L'unité de l'ensemble est assurée par les répétitions et les reprises anaphoriques.

14 Avec la thématisation d'un circonstant adverbial de lieu, le paragraphe 225 introduit une halte dans le récit:c'est alors le rhème qui prend le pas sur le thème, avec la rhématisation du reste de l'énoncé. Pause qui s'étend sur les deux paragraphes 225 et 226: de l'action, on passe aux conséquences et à la parole. Pause de courte durée: le paragraphe 227, hâché, de rupture en retour, relance l'engagement dans le désordre, jusqu'à sa conclusion.

15 Ainsi, les thématisations apparaissent comme un outil de structuration du texte, d'organisation et de hiérarchisation du récit. Par ailleurs, à l'intérieur du cadre chronologique fixé par la thématisation de circonstants temporels, et parallèlement aux épisodes ainsi délimités, le texte se construit selon d'autres thématisations: l'ordre thème-rhème coïncide avec l'ordre canonique de la phrase ; c'est un nouveau type de séquences, une succession de gros plans sur les personnages, qui priment sur les événements. Quelques exemples :

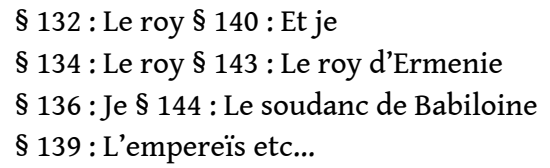

Personnages principaux (le roy), personnages secondaires (messages, freres prescheurs) sur lesquels peut s'attarder la caméra, par le jeu des progressions et des reprises anaphoriques; ainsi au paragraphe 134 :

le roy reçut moult debonnairement ses messages, [...]

Et par les messages envoia le roy au roy des Tartarins une tente faite en la guise d'une chapelle [...]

et le roy [...] fist entailler en ladite chapelle par ymages l'Anonciacion Nostre Dame et touz les autres poins de la foy.

Et ces choses leur envoia il par.II. Freres prescheurs [...]

Si revindrent au roi les .II. freres [...]

Ou encore, § 144 :

Le soudanc de Babiloine [...] s'apensa que il iroit confonder le soudanc de Hamant [...]

Le soudanc de Hamant [...] fit tant barguigner au ferrais le soudanc de Babiloine que le

ferrais l'empoisonna

Et la maniere de l'empoisonnement ...

L'organisation interne de ces séquences de «personnages » repose ainsi, sporadiquement, sur des progressions linéaires. Mais la juxtaposition d'énoncés en rupture thématique est le procédé le plus fréquent - la cohésion du texte est alors assurée par les reprises anaphoriques.

19 La thématisation du sujet délimite donc des plages textuelles centrées sur un personnage (ou un groupe) dominant; alternant avec le cadrage thématique des circonstants, elle intègre les personnages dans la chronologie. L'histoire se construit suivant une double dimension, temporelle et spatiale, par la succession de séquences datées et de gros plans. 
Les personnages ainsi isolés par la thématisation sont nombreux, centraux ou épisodiques, récurrents ou singuliers. De cet ensemble se détachent les deux protagonistes : le roi et Joinville.

21 Le roi apparaît dans la plupart des phrases du texte, en position thématique ou rhématique. Ainsi, entre les paragraphes 146 et 151, on relève 27 occurrences, y compris les substituts pronominaux et sans compter les déterminants possessifs. n'apparaît que 4 fois en position de thème, confondu avec le sujet de la phrase c'est le cas de figure analysé ci-dessus : sur un fond de séquences chronologiquement organisées et enchaînées, qui constituent le cadre événementiel où se mêlent et se côtoient personnages, comparses et figurants (le roy et les barons et les autres pelerins), qui plantent le décor et enchaînent des plans d'ensemble, se détache alors le personnage central - le roy - en gros plan.

Mais il y a plus. Ainsi, au paragraphe 146, la première phrase - Maintenant que mars entra, par le commandement le roy, le roy et les barons et les autres pelerins commanderent que les nez refeussent chargees de vin et de viandes pour mouvoir quant le roy le commanderoit - comporte une structure thématique complexe. Si le cadrage est assuré par la thématisation d'un circonstant temporel (l'ensemble de la phrase apporte une information relative a des faits ainsi datés), il est difficile de considérer que la suite de la phrase en constitue en bloc le rhème, les différents constituants ayant des valeurs différentes sur l'échelle du dynamisme communicatif. Le second groupe déplacé, par le commandement le roy, est, lui aussi, en position thématique : le roy intervient donc indirectement comme thème de l'énoncé. Et au-delà ? De quoi, de qui parle-t-on, sinon du roy? Présent explicitement trois fois dans la phrase, ici en position de sujet, on peut dire qu'il constitue le thème propre d'une phrase dans laquelle les circonstants antéposés sont les thèmes secondaires. Dans l'extrait cité comme dans l'ensemble du texte de Joinville, le roy est le thème dominant.

146 Maintenant que mars entra, par le commandement le roy, le roy et les barons et les autres pelerins commanderent que les nez refeussent chargees de vin et de viandes pour mouvoir quant le roy le commanderoit. Dont il avint ainsi que quant la chose fu bien aree, le roy et la royne se requeuillirent en leur nez, le vendredi devant Penthecouste, et dist le roy a ses barons que il alassent après li en leur nez droit vers Egypte. Le samedi fist le roy voille et touz les autres vessiaus aussi, qui fut moult belle chose a veoir, car il sembloit que toutez la mer tant comme l'en pooit veoir a l'ueil feust couverte de touailles et voilles des vessiaus, qui furent nombrez a .XVIII'. vessiaus, que granz que petiz. 147 Le roy ancra au bout d'un tertre que l'en appele la pointe de Limeson, et touz les autres vessiaus entour li. Le roy descendi a terre le jour de la Pentecouste. Quant nous eumes oÿ la messe, un vent grief et fort qui venoit de vers Egypte leva en tel maniere que de .II. mille et .VIII ${ }^{c}$. chevaliers que le roy mena en Egypte, ne l'en demoura que.VII. cens que le vent ne les eust dessevrés de la compaignie le roy, et menez en Acre et en autres terres estranges, qui puis ne revindrent au roy de grant piece.

148 L'andemain de la Penthecouste, le vent fu cheu. Le roy, et nous qui estions avec li demourez, si comme Dieu voult, feismes voille. De rechief et encontrames le prince de la Moree et le duc d Bourgoingne, qui avoit sejourné en la Moree. Le jeudi après Penthecouste ariva le roy devant Damiete ; et trouvames la trestout le pooir du soudanc sur la rive de la mer, moult beles gent a regarder, car le soudanc porte les 
armes d'or, la ou le soleil feroit, qui fesoit les armes resplendir. La noise qui il menoient de leur nacaires et de leus cors sarrazinois estoit espouentable a escouter.

149 Le roy manda ses barons pour avoir conseil que il feroit. Moult de gens li loerent que il attendit tant que ses gens feussent revenus, pour ce que il ne li estoit pas demouré la tierce partie de ses gens et il ne les en voult onques croire, la reson pour quoy que il dit que il en donroit cuer a ses ennemis, et meismement que en la mer devant Damiete n'a point de port la ou il peut sa gent attendre, pour ce que un fort vent nes preist et les menast en autres terres aussi comme les autres avoient le jour de Penthecouste.

150 Acordé fu que le roy decendroit a terre le vendredi devant la Trinité et iroit combattre aus Sarrazins se en eulz ne demouroit. Le roy commanda a mon seigneur Jehan de Baumont que il feist bailler une galie a mon seigneur Erart de Brienne et a moy pour nous descendre et nos chevaliers, pour ce que les grans nefz n'avoient pooir de venir jusques a terre. 151 Aussi comme Diex voult, quant je reving a ma nef, je trouvai une petite nef que ma dame de Baruch, qui estoit cousine germainne le conte de Monbeliart et la nostre, l'avoit donnee, la ou il avoit.VIII. de mes chevaus. Quant vint au vendredi, entre moy et mon seigneur Erart touz armés alames au roy pour la galie demander, dont mon seigneur Jehan de Biaumont nous respondi que nous n'en arions point.

\section{Joinville narrateur : « Discours » ou « Histoire »?}

Le roi ou Joinville? Nombreux sont les fragments du texte pour lesquels, au terme d'une analyse semblable, on montrerait que c'est le «Je / Nous" qui est le thème dominant. Alors « Vie de Saint Louis » ou « Vie de Joinville »? Biographie ou autobiographie ? Point n'est besoin de nous étendre sur le contenu réferentiel de ce nous, qui évidemment contient un je, de dénombrer statistiquement les occurrences respectives de ce je et du roy , ni même de départager les emplois de l'un et de l'autre en position thématique. Le personnage central de la Vie de Saint Louis est bien Saint Louis, le roi. Mais le roi n'est pas «celui dont on parle», il est «celui dont parle Joinville». Au Je / nous omniprésent s'ajoutent d'autres marques énonciatives, d'autres expressions de la subjectivité qui font de la Vie de Saint Louis un récit dans lequel les faits ne semblent pas se dire d'eux-mêmes, un récit entièrement assumé par l'énonciation discursive, et fortement modalisé.

Sans doute ne faut-il pas s'étonner de trouver tout au long du récit des termes évaluatifs qui célèbrent la vaillance des chevaliers croisés et leurs faits d'armes. Ainsi, entre autres :

§ 154 : II. moult vaillans chevaliers

\$ 197 : Les Templiers, qui estoient venus au cri, firent l'ariere garde bien et hardiement

$\S 229$ : Et sachiés que ce fut un biau fait d'armes

$\S 273$ : Le conte de Flandres et sa bataille coururent sus moult aigrement et viguereusement et a pié et a cheval...

La se prouva viguereusement Gautier de la Horgne...

§ 276 :... mon seigneur Henri de Cooigne, qui estoit en l'ost le duc de Bourgoingne, sage chevalier et preus et apensé...

Louange aux preux chevaliers, hommage à l'adversaire :

\$199: Son nom estoit Secedin, le fils seic, ce vaut autant a dire comme "le veel, le filz au veel». Son nom tenoient il a moult grant chose en la paiennime, car ce sont les gens ou 
monde qui plus honneurent gens anciennes, puis que il est ainsi que Dieu les a gardés de vilain reproche jusques en leur vieillesce. Secedin, ce vaillant Turc.... dans l'ensemble de la Vie de Saint Louis, de telles formulations sont relativement rares et n'interviennent qu'à bon escient. Elles font écho à d'autres expressions axiologiques, comme :

§ 146 : le samedi fist le roy voille et touz les autres vessaius aussi, qui moult fu belle chose a voir ...

$\S 148$ : et trouvames la trestout le pooir du soudanc sur la rive de la mer, moult beles gent

a regarder

ou encore, selon le jugement du temps :

$\S 252$ : De touailles sont entorteillees leur testes, qui leur vont par desous le menton, dont ledes gent et hydeuses sont a regarder, car les cheveux des testes et des barbes sont touz noirs.

§ 253 : J'ai veu en cest païs, puis que je revins d'outre mer, aucuns desloiaus crestiens qui tenoient la loy des Beduyns...

etc.

Ces expressions parsèment un récit-témoignage « impressionniste », dans lequel on note en particulier nombre d'occurrences du verbe sembler, ou des formules comme au § 222 Nous trouvames bien.VIM. Turs, par esme, qui n'engagent que la responsabilité de l'auteur !

29 A l'historien de découvrir les faits derrière ce témoignage constamment avoué d'un narrateur juge de ses pairs - $\S 170$ : les gens le roy, qui deussent debonnerement les gens retenir, leur loerent les estaus pour vendre leur danrees aussi chiers... qu'ils le purent ou de ses adversaires - § 164: Mal apertement se partirent les Turs de Damiete...; § 191 : et toute la puissance du soudan se logerent sur le fleuve de Rexi, d'autre part par devant nostre ost pour nous deffendre le passage, la quele chose leur estoit legiere car nulz ne pooit passer la dite yaue par devers eulz se nous ne la passions a nou.

Certes, l'écart entre le temps de l'action et le temps de l'écriture expliquerait cette distanciation, la transposition du fait en mémoire, de l'Histoire en Discours. Joinville acteur, juge et témoin. Car ce Je omniprésent s'adresse à un tu tout aussi omniprésent.

«Adresses » explicites au lecteur :

$\S 157$ : Et soiés certain que ...

$\S 160$ : Je vous avoie oublié a dire...

$\S 166$ : ....et comment nous l'oubliames vous diré je ci après

$\S 173$ :...les preudeshomes chevaliers qui estoient avec le roy vous ai je ramenteu...

$\S 213$ :... nous eussions le soir gueté en grant peril, aussi comme nous eussiens fait a l'autre

guiet dont je vous ai parlé avant

$\S 308$ : Or vous lerai ici, si vous dirai comment le roy fu pris ...

Souci « pédagogique » d'être suivi et compris, parfois explicité - $\$ 280$ : pour ce que il nous convient poursuivre nostre matiere, la quele il nous couvient un pou entrelacier pour faire entendre comment les soudanc tenoient leur gens ordeneement et areement...-, qui se manifeste par l'intrusion d'éléments discursifs en marge du récit : rappels et retours en arrière comme aux $\S \S 160$ et 213 , cités ci-dessus, ou encore au $\S 246$ : En celle bataille ot moult de gent, et de grant bobant, qui s'en vindrent moult honteusement fuiant parmi le poncel dont je vous ai avant parlé.; commentaires et explications : \$142, par exemple, sur un mot évocateur d'une réalité « exotique »: « Ferrais » est cil qui tient les paveillons au soudanc et qui li nettoie ses mesons. 
Ainsi se justifient, par exemple, les longues «digressions » sur le Nil, les Bédouins, l'évocation du feu grégeois, passages informatifs qui ralentissent mais étoffent la narration; rompant l'enchainement linéaire des séquences d'action, ces séquences adventices, en «flash-ahead $»^{4}$, en font l'arrière plan, leur donnent réalité et profondeur, et entrelacent au discours de l'histoire le discours du témoignage, de l'expérience vécue. C'est cette double énonciation discursive qui construit le récit de la croisade d'Egypte, et le texte de la Vie de Saint Louis dans son ensemble. L'introduction, d'ailleurs, l'annonce (§ 6): Le second livre vous parlera de ses granz chevaleries et de ses granz hardemens, les quiex sont tiex que je li vi quatre foiz mettre son cors en aventure de mort, aussi com vous orrez ci après, pour espargnier le dommage de son peuple.

\section{Joinville témoin : suprématie de la parole}

Dans l'Avant-propos à son édition de la Vie de Saint Louis ${ }^{5}$, Jacques Monfrin écrit (pp.II et III) : « l'allure générale est celle du récit oral. Joinville avait dû raconter bien souvent ses souvenirs; il ne change pas de ton lorsqu'il s'agit de les mettre par écrit, qu'il les ait dictés, ce qui est de beaucoup le plus vraisemblable, ou qu'il les ait écrits, ce dont il était certainement capable.»

Nul ne contestera ce caractère d'oralité, affirmée par l'auteur tout au long de ce « Dit de Saint Louis "; en écho aux adresses directes au lecteur/auditeur, l'emploi, fréquent, du verbe dire (ou de verbes de parole) à la $1^{\text {re }}$ personne confirme le caractère discursif du texte. Nous n'en citons que quelques exemples:

$\S 135$ : Comment les messages le roy de France furent receus vous diréje...

$\$ 160$ : Je vous avoie oublié à dire que...

$\$ 165$ : Or disons donc que grant grace nous fist Dieu...

\$167: Je vous prenré premierement au roy...

$\S 191$ : Or revenons a nostre premiere matiere et disons ainsi que...

$\$ 194$ : ...aussi comme je vous ai dit devant...

$\$ 266$ : Tout premier je vous dirai du roy de Sezile...

etc.

Joinville, témoin-acteur, réclame ainsi l'adhésion à son récit. Mais au-delà, son dire est constamment nourri et se construit de « dires autres »: les discours rapportés abondent sous toutes les formes, avec des statuts différents.

$\S 199$ : Secedin, ce vaillant Turc, aussi comme les espies le roy le raporterent, se vanta que il mangeroit le jour de la fete saint Sebastien es paveillons le roy.

$\S 219$ : Le Temple, ainsi comme le maistre le me dit depuis, y perdi .XIIIIXX. homes armés, et touz a cheval.

La « modalisation par discours autre », qui « renvoie l'assertion à un autre discours $»^{6}$, est employée ici «en négatif » : paradoxalement, cette distanciation du locuteur par rapport à son dire ne traduit pas son désengagement mais confirme son assertion et doit entraîner une adhésion accrue du lecteur-auditeur.

Ainsi, d'ailleurs, s'expliquent et se justifient aussi ces « appels à témoin » qui parsèment l'œuvre, au discours indirect :

$\S 236$ : Et dit l'en que nous estions trestous perdus...

$\S 289$ : ... et dit l'en que c'estoit pour ce que les fielz en estoient pourriz. ou en « négatif » encore : 
$\S 248$ : Ne je n'ö̈ onques dire que les Beduyns ...

§ 290 : Ne onques n'oÿ dire que nulz n'y feust retrouvez... : l'absence de témoin est, elle aussi, preuve et garante de vérité.

La modalisation par discours autre, au niveau du contenu est donc fondamentalement constitutive du discours de Joinville. Elle est à distinguer d'une autre forme de modalisation autonymique ${ }^{7}$ qui renvoie à des mot «venus d'ailleurs » en marge de la narration; nous l'avons vu, c'est le discours du commentaire et de l'explication :

$\S 173$ : et tiex chevaliers soloit l'en appeler bons chevalier.

$\S 203$ : un engin que l'en appele perriere

ou encore $\S 142$ : «Ferrais » est cil qui tient les paveillons au soudanc ...

A côté de ces faits, constants mais épars, le discours rapporté est omniprésent. Dans ce « compte-rendu » de la croisade d'Egypte se mêlent intimement, et à part égale, la parole et l'action. Mais le mélange n'est ni gratuit ni aléatoire ! Il est orchestré et organisé sur plusieurs plans.

1 En premier lieu, tout au long du texte, la parole fait partie de l'action : action de guerre, qui implique ordres, requêtes, conseils, ambassades...

$\S 146$ : le roy et les barons et les autres pelerins commanderent que les nez refeussent chargiees [...] et dist a ses barons le roy que il alassent apres li en leur nez droit vers l'Egypte

$\S 157$ : Mon seigneur Baudoin de Reins [...] me manda par son escuier que je l'attendisse...

$\S 178$ : Et pour ce ordena le roy que les batailles qui soloient guietier a cheval guieteroient a pié.

Ce sont en général des discours indirects brefs (une phrase enchâssée); les verbes introducteurs sont en nombre limité et maintes fois repris : dire, mander, commander, loer, requerre, demander, répondre, escrier..., tous verbes de communication « objectifs ", exempts de toute modalisation. Ce qui est ici rapporté, c'est une parole désincarnée, le squelette d'un discours autre ramené à sa signification globale, intégré dans le cadre énonciatif, les mots et la continuité du discours rapportant, balisé par le verbe introducteur et, éventuellement, le mode de la subordonnée.

Ces discours là sont à une ou deux voix. Mais il arrive aussi que les échanges se poursuivent :

$\S 152$ : Je demandai au mestre combien il i avoit trop de gens et il me dist «Vingt hommes a armes ». Et si li demandai se il menroit bien nostre gent a terre [...]; et il me respondi: «Ö̈l».

Il arrive que les voix se multiplient et que le discours rapporté se complexifie :

$\S 183$ : ...le roy manda touz ses barons de l'ost pour savoir quel voie il tendroit [...]

Dont il avint ainsi que le bon conte Pierre de Bretaingne et le plus des barons de l'ost s'acorderent que le roy alast assieger Alixandre, pour ce que devant la ville avoit bon port, la ou les nez arriveroient qui aporteroient les viandes en l'ost. A ce fu le conte d'Artois contraire, et dit ainsi que il ne s'acorderoit ja que en alast mais que en Babiloine pour ce que c'estoit le chief de tout le royaume d'Egypte; et dit ainsi que qui vouloit tuer la serpent, il li devoit premier esquacher le chief.

Si chacun des discours ainsi rapportés est, comme les précédents, un maillon dans l'enchaînement des faits, les plages discursives constituées par ces échanges ont un statut spécial et jouent un rôle particulier dans l'organisation et la structuration du texte. Nous en donnons un exemple. 
$\mathrm{Au}$ paragraphe 220 s'ouvre une longue séquence d'action: Moy et mes chevaliers acordames que nous irions sus courre a pluseurs Turs qui chargoient leur harnois a main senestre en leur ost et leur courumes sus.

Les paragraphes suivants déroulent la bataille dans une alternance de plans d'ensemble ( Moy et mes chevaliers, il, les Turs, une grant route de Turs...) et de gros plans isolant de la multitude combattante les protagonistes du combat, les héros de la croisade :

- gros plan narratif, quelquefois, comme dans ce corps à corps sur fond de bataille (§§ 220-221) : Endementres que nous les chacions parmi l'ost, je regardai un Sarrazin qui montoit sur son cheval; un sien chevalier li tenoit le frain. La ou il tenoit ses.II. mains a sa selle pour monter, je li donné de mon glaive par desous les esseles et le getai mort [...].

- gros plan discursif le plus souvent: de longues plages de discours rapportés, direct et indirect, rompent le rythme de l'action, halte provisoire ( $\$ 226$, cité ci-dessus p. 306) ou changement de cap (\$227, ibid.).

Les paragraphes 230 à 234 en sont une autre illustration. Ici encore, sur fond de bataille (§ 231 : Les serjans les alerent querre en la bataille, ou le hutin estoit grant d'eulz et des Turs. A l'esmouvoir l'ost le roy rot grant noise de trompes et de nacaires et de cors sarrazinnois), se détache et se rassemble autour du roy le groupe des preudommes chevaliers de son conseil (§ 232).

C'est incontestablement le discours indirect qui domine dans ces exemples comme dans l'ensemble du texte; mais le discours direct se trouve aussi, sporadiquement. Volontairement ou arbitrairement?

Il semblerait, à l'examen des occurrences, - le statut autonyme de l'énoncé représenté dans le discours direct y conduit - que Joinville ait joué volontairement sur cette opposition entre l'homogénéité du discours indirect et l'hétérogénéité du discours direct. Hétérogénéité occasionnelle, lorsque la forme du discours initial interdit le rapport indirect : c'est le cas de l'exclamation (au § 237 par exemple : il disoit moult souvent : « Voi ! pour le chief Dieu, avez veu ces ribaus!", ce fait annexe n'appelant pas nécessairement l'adhésion du rapporteur) ou encore de la prière, elle aussi dans une certaine mesure en marge du récit (\$ 225). Hétérogénéité paradoxale dans ce cas, très voisin de ce que l'on appelle « discours indirect avec îlot textuel »: Nous venimes a un poncel qui estoit parmi le ru, et je dis au connestable que nous demourissons pour garder ce poncel, "car se nous le lesson il ferront sus le roy par deça; et se nostre gnet sont assaillis de.II. pars il pourront bien perdre » (§ 235). Ici le rapporteur, rapportant ses propres paroles comme explicitation de l'assertion rapportée indirectement, souligne son adhésion totale à son propre discours, dont il accroît la force persuasive. Hétérogénéité recherchée, comme au paragraphe 226, où le discours direct isole les deux premières « répliques » de la suite de l'échange et du même coup centre la caméra sur deux protagonistes.

Parlerons-nous, enfin, du rôle conclusif du discours direct? § 233: Et le roy dit: "Connestable, alés devant, et je vous suivré. ». C'est le dernier mot de l'échange, la fin du conseil au discours indirect. § 234 : Lors dis je au connestable: "Sire, nous n'avon pooir d'aller au roy parmi ceste gent; maiz alons amont et metons cest fossé que vous veez devant vous entre nous et eulz; et ainsi pourrons revenir au roy. " C'est la fin de l'épisode.

2 Ainsi, la structuration du texte, que révèle le jeu des constructions thématiques, repose pour une large part sur l'alternance entre les deux voix du discours rapporteur et des discours rapportés et le jeu subtil de l'opposition entre discours indirect et discours direct. 
Graphie ou autobiographie ? Est-ce bien là le vrai débat ? « La manière dont le livre est construit surprend parfois, mais Joinville est moins désordonné qu'on ne l'a dit» (J. Monfrin, loc. cit., p.I). Désordonné, Joinville? La Vie de Saint Louis offre au contraire l'exemple d'un texte élaboré, dont la construction révèle une grande maîtrise des techniques d'écriture et de composition de son auteur.

230 Endementres que nous estiens ainsi, mon seigneur Jehan de Waleri, le preudome, vint au roy et li dit que il looit que il se traisist a main destre sur le flum, pour avoir l'aide du duc de Bourgoingne et des autres qui gardoient l'ost que nous avions lessié, et pour ce que ses serjans eussent a boire, car la chaut estoit ja grant levé. 231 Le roy commanda a ses serjans que il li alassent querre ses bons chevaliers que il avoit entour li de son conseil, et les nomma touz par leur non. Les serjans les alerent querre en la bataille, ou le hutin estoit grant d'eulz et des Turs. Il vindrent au roy, et leur demanda conseil ; et il distrent que mon seigneur Jehan de Waleri le conseilloit moult bien. Et lors commanda le roy au gonfanon saint Denis et a ses banieres qu'il se traisissent a main destre vers le flum. A l'esmouvoir l'ost le roy rot grant noise de trompes et de nacaires et de cors sarrazinnois. 232 Il n'ot guieres alé quant il ot pluseurs messages du conte de Poitiers, son frere, du conte de Flandres et de pluseurs riches homes qui illec avoient leur batailles, qui touz li prioient que il ne se meust, car il estoient si pressé des Turs que il ne le pooient suivre. Le roy rapella touz ses preudomes chevaliers de son conseil, et touz li loerent que il attendit. Et un pou après mon seigneur Jehan de Waleri revint qui blasma le roy et son conseil de ce que il estoient en demeure ; après tout son conseil li loa que il se traisist sur le flum aussi comme le sire de Waleri li avoit loé. 233 Et maintenant le connestable mon seigneur Hymbert de Biaujeu, vint a li et li dit que le conte d'Artois, son frere, se deffendoit en une meson a la Massourre et que il l'alast secourre. Et le roy li dit : «Connestable, alés devant, et je vous suivré. » Et je dis au connestable que je seroie son chevalier, et il m'en mercia molt. Nous nous meismes a la voie pour aler a la Massourre. 234 Lors vint un serjant a mace au connestable tout effraé, et li dit que le roy estoit aresté et les Turs s'estoient mis entre li et nous. Nous nous tornames et veimes que il en avoit bien mil et plus entre li et nous, et nous n'estions que .VI. Lors dis je au connestable : « Sire, nous n'avon pooir d'aller au roy parmi ceste gent; maiz alons amont et metons cest fossé que vous veez devant vous entre nous et eulz; et ainsi pourrons revenir au roy. » Ainsi comme je le louai le connestable le fist. Et sachiez que se il se feussent pris garde de nous, il nous eussent touz mors; mez il entendoient au roy et aus autres grosses batailles, par quoy il cuidoient que nous feusson des leur.

Maîtrise d'autant plus grande que l'œuvre ne suit pas les modèles du genre. CEuvre historique, ancrée dans la chronologie, elle n'est pas seulement une chronique : dans un cadre événementiel, fixé dans le texte par la thématisation des circonstants temporels, vivent et agissent les personnages : le roy... et les preudeshomes chevaliers qui estoient avec le roy (\$173), parmi lesquels Joinville. Ce sont eux qui ont fait l'histoire, que Joinville déroule non comme un récit mais comme une action. Fragment de la Vie de Saint Louis, le récit de la campagne d'Egypte n'est extrait ni d'une biographie ni d'un récit autobiographique; c'est une œuvre qui tient de la chronique et de l'épopée, une œuvre dramatique qui transcende l'expression d'une réalité vécue. L'écriture met en évidence le 
caractère scénique - pour ne pas dire cinématographique - de cette œuvre «moderne » d'une grande valeur littéraire.

\section{NOTES}

1. Le Goff J., Saint Louis, Paris, Gallimard, 1996, p.16.

2. Zink M., La Subjectivité littéraire. Autour du siècle de Saint Louis, Paris, 1985, p.219.

3. Le Goff J., op.cit., p.479.

4. sur le modèle de flash-back, terme utilisé par J.-J. Annaud pour désigner des séquences en rupture, projetées vers l'avenir ou hors de l'enchaînement chronologique.

5. Garnier Flammarion, Paris, 1995.

6. Voir en particulier, Jacqueline Authier-Revuz, « Modalisation par discours autre et bivocalité », in R. Tomassone (dir.), Une langue : le français, Paris, Hachette, 2001, pp.200-201.

7. «qui consiste à reconnaître dans les mots dont on fait usage, leur caractère 'venu d'ailleurs'» (ibid., p.201).

\section{AUTEUR}

\section{ROBERTE TOMASSONE}

I.U.F.M. de Versailles - Paris 10 